\title{
Anatomy of the anteromedial thigh flap based on the oblique branch of the descending branch of the lateral circumflex femoral artery
}

\author{
V. Tayfur, O. Magden, M. Edizer, G.N. Yonguc, F. Aksu, N. Gocmen-Mas \\ Department of Anatomy, Faculty of Medicine, Dokuz Eylul University, Izmir, Turkey \\ [Received: 24 February 2015; Accepted: 3 September 2015]
}

\begin{abstract}
Anteromedial thigh (AMT) flaps based on lateral circumflex femoral artery (LCFA) have characteristics which make them favourable for use in reconstruction of extensive thigh, head, neck and leg defects. AMT flap which is elevated on the artery has the advantages of low donor site morbidity and preservation of main arteries. Due to inconstant anatomy of the pedicle, the flap is mostly not preferable. Hence, we aimed to describe the anatomical features of the unnamed branch of the descending branch of the LCFA harvesting AMT flap. For this purpose, the external iliac artery was displayed bilaterally on 15 adult $(13$ males and 2 females; age range 55-82 years) preserved cadavers using latex injection. The perforator branch of the descending branch from the LCFA was microdissected under $4 \times$ loupe magnification. The perforator branch was located $28.53(20.20-34.20) \mathrm{cm}$ distal to the anterior superior iliac spine, $22.12(13.40-28.00) \mathrm{cm}$ distal to the pubic tubercle, and $13.20(10.80-16.20) \mathrm{cm}$ proximal to the interepicondylar line. At the level of origin point the mean diameter of the perforating branch was $0.17 \mathrm{~cm}$ and the mean diameter of its cutaneous branch was $0.14 \mathrm{~cm}$. The mean length of the pedicle was $5.71(3.70-9.00) \mathrm{cm}$. We conclude that our findings contribute to the literature in terms of anatomical knowledge for surgical safety. (Folia Morphol 2016; 75, 1: 101-106)
\end{abstract}

Key words: anteromedial thigh flap, lateral circumflex femoral artery, unnamed branch, perforator, pedicle

\section{INTRODUCTION}

Anteromedial fasciocutaneous flaps from the thigh region based on the lateral circumflex femoral artery (LCFA) are important for repairing the defects of wide inguinal scars, the soft tissue defects following foot injury, after benign or extensive malignant tumour ablation of head and neck region, lower abdomen, leg, major scrotal defects, post-burn contractures in the body $[1,10,12,19,31]$.

Classically, the precise vascular basis of fasciocutaneous flaps is not exactly known [18]. Additionally, there is terminological confusion in literature on the branching pattern, and also nomenclature of the flap's vascular anatomy. According to recent findings, the anteromedial flaps are usually based on the branches of the LCFA $[4,5,27]$. The artery also possesses 3 branches: the ascending, the transverse, and the descending [26]. A directly innominate branch which arises from the descending branch of the LCFA runs posteromedially to the rectus femoris muscle and the vastus medialis. The unnamed branch which has been named as oblique branch by Cigna et al. [4], gives

Address for correspondence: Ass. Prof. N. Gocmen-Mas, MD, PhD, Dokuz Eylul University, Faculty of Medicine, Department of Anatomy, Inciralti Izmir, Turkey, tel: +90 3123046043, e-mail: nuket.mas@deu.edu.tr; nuketmas@gmail.com 
several muscular branches (MBs) to both the sartorius and the vastus medialis muscles. Then, it traverses as the septocutaneous branch which emerges at the junction of the rectus femoris, sartorius and vastus medialis muscles and supplies overlying skin as the fasciocutaneous branch $[5,27]$.

A point in a small triangle at approximately midthigh represents the juncture of the medial margin of the rectus femoris, lateral border of the sartorius, and the underlying vastus medialis muscles, which is marked as an indicating point where septocutaneous perforator branch (PB) appears. The perforating and cutaneous branches (CBS) of LCFA are important in terms of fasciocutaneous anteromedial flap surgery, elevated from thigh due to its rich and convenient supply. The anteromedial thigh (AMT) flap has been considered as less preferable than the anterolateral thigh flap due to its variability [5]. We aimed to solve the ambiguity on nomenclature, origin, localisation, length, size and adjacent relationships of the flap pedicle. We believe that the results may contribute to knowledge on surgical procedures in cases with inguinal, perineal, leg, head and neck defect and ways of providing easy anastomose normal anatomical reorganisation and easing the operative period.

In the present study, vascular anatomical features of the AMT flap, which is based on the LCFA, were evaluated to find possible vascular variants for this region to ensure a reliable flap surgery.

\section{MATERIALS AND METHODS}

The first important step of perforator flap dissection is to determine the location of the perforating branch.

Dissections of 15 adults (13 males, 2 females; age range $55-82$ years: bilaterally 30 cases) formalin fixed preserved cadavers were performed under $4 \times$ magnification with a lupe. The measurements were taken using a fine microcalliper and were expressed in centimetres. After elevating the anteromedial fasciocutaneous flap, meticulous micro dissections of the thigh region were performed bilaterally. The external iliac arteries were identified by injecting coloured latex. The point of origin of the LCFA was observed from the deep femoral artery. The ascending, transverse and descending branches of LCFA were displayed. The PBs that supply the AMT flap were detected. The MB and $\mathrm{CB}$ of the $\mathrm{PB}$ were also displayed by performing tangential dissection of the skin, subcutaneous fat tissue, fascia and muscular layers.
The following parameters were evaluated: (1) the location, course and the diameter at origin and length of the PB; (2) the location and course; the diameter at origin and length of the MB to sartorius muscle; (3) the location and course; the diameter at origin and length of the $C B$; distance between the origin point of the $\mathrm{PB}(4)$ and the anterior superior iliac spine (ASIS); (5) and the pubic tubercle; (6) and also the interepicondylar line, respectively; (7) the length of the pedicle; features of the accompanied (8) veins and (9) nerve were noted in all of the cases. The length of $C B$ was accepted as pedicle length of the AMT flap, as $C B$ ends on the overlying skin.

\section{RESULTS}

The dissections of LCFA were carried out in 30 cases bilaterally ( 13 males, 2 females; age range 55-82 years). The artery arose from the deep femoral artery in all of the cases.

Only one PB supplying the AMT flap was detected for each of the cadavers. The PB originated from the descending branch of LCFA. In literature, the PB has been named as the innominate branch [25], medial branch [30] or oblique branch [4]. Based on our observations during dissections we thought of naming this unnamed branch as the oblique branch due to its oblique course as described by Cigna et al. [4]. We observed that in all of the cases PB exits laterally to the sartorius muscle at approximately within the small triangle that was formed by the sartorius, rectus femoris and vastus medialis muscles (Figs. 1, 2). The anatomical features of the $\mathrm{PB}, \mathrm{MB}$ (to sartorius muscle) and $C B$ are presented in Table 1. The perforating branch originated from the descending branch of LCFA, and appeared to be between the middle and lower part of the thigh. PB was accompanied by two innominate cutaneous veins and branches of the anteromedial cutaneous nerve in all of the cases.

\section{DISCUSSION}

AMT flaps based on the PBs of LCFA have been used in local or wide skin defect reconstructions. These flaps have been especially preferred to repair benign or malignant scrotal and vulva-vaginal tumour excision or radiotherapy related defects, trauma scar contractures of thigh, lower leg and foot defects, urethral reconstructions in epispadias and hypospadias, local and extensive defects of the head and the neck region $[6,9,10,14,17,31]$. 


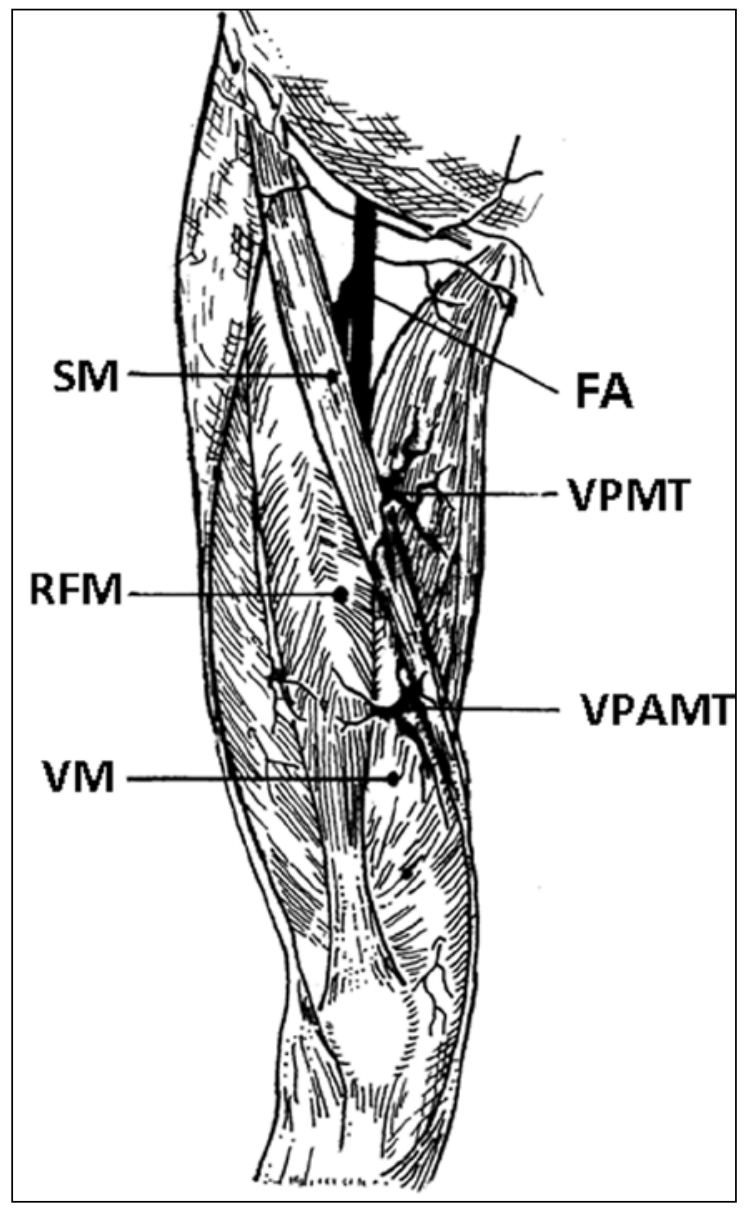

Figure 1. Anterior aspect of thigh showing the principal branches to the skin; FA — femoral artery; VPMT — vascular pedicle of the medial thigh fasciocutaneous flap; VPAMT — vascular pedicle of the anteromedial thigh fasciocutaneous flap; VM — vastus medialis muscle; RFM — rectus femoris muscle; $\mathrm{SM}$ — sartorius muscle.

There are limited number of studies contributing to the definition of the vascular anatomy of the LCFA system as the AMT flap pedicle $[2,4,7,9,13,15$,

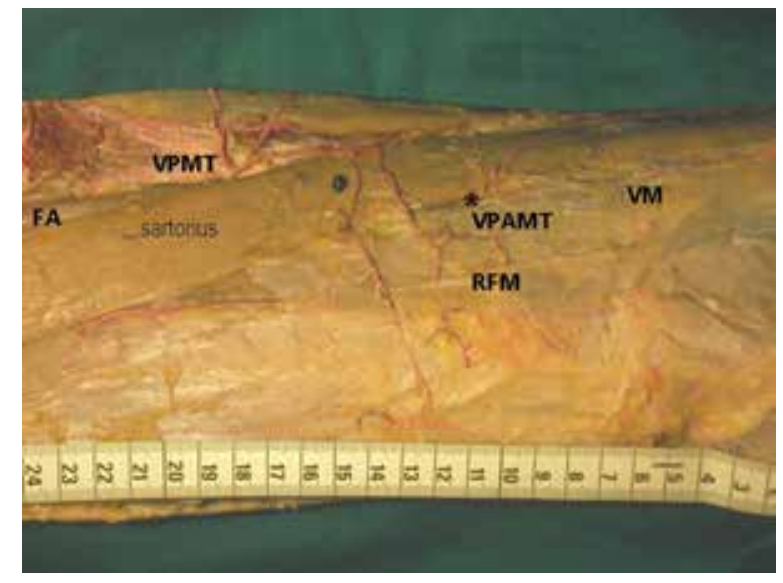

Figure 2. Branches of the femoral artery (FA) were shown emerging along the borders of sartorius muscle to supply the skin; *VPAMT — vascular pedicle of the anteromedial thigh fasciocutaneous flap; VPMT - vascular pedicle of the medial thigh fasciocutaneous flap; VM — vastus medialis muscle; RFM — rectus femoris muscle.

$16,23,24,28-30]$. Thus, anatomy of the AMT flap is not yet clear. Perforator flaps of the anterior thigh region with different vascular sources were encountered under the name of AMT [29]. Hence, a clear and extensive classification is needed for the AMT flaps to prevent confusion. Besides the anatomic variants, the perforator direction and the vessels of the pedicle were quite permanent. According to the terminology rules for perforator flaps, we aimed to identify the AMT flap pedicle based on the source vessel as LCFA and on the muscle pierced $[3,8]$. The flap was firstly described by Song et al. [26] naming the source artery as the perforator, the innominate branch of LCFA. Some authors referred to the flap described by Song et al. [26] and called the branch the medial descending branch of LCFA, the rectus femoris branch, the rectus femoris artery, the in-

Table 1. The data obtained from the cadaver dissections

\begin{tabular}{lccc}
\hline & Mean [cm] & Minimum [cm] & Maximum [cm] \\
\hline Diameter of PB (at origin) & 0.17 & 0.12 & 0.21 \\
Distance between piercing point of PB and ASIS & 28.53 & 20.20 & 34.20 \\
Distance between piercing point of PB and IL & 13.20 & 10.8 & 16.20 \\
Distance between piercing point of PB and PT & 22.12 & 13.40 & 28.00 \\
Diameter of CB (at origin) & 0.14 & 0.10 & 0.19 \\
Length of CB (pedicle length) & 5.71 & 3.70 & 9.00 \\
Diameter of MB (at origin) & 0.08 & 0.05 & 0.11 \\
Length of MB & 1.40 & 1.10 & 1.70 \\
\hline
\end{tabular}

CB — cutaneous branch; PB — perforator branch; MB — muscular branch to sartorius muscle; ASIS — anterior superior iliac spine; IL — interepicondylar line; PT — pubic tubercle 
nominate branch, the oblique branch which does not permanently give off perforators $[4,25,29,30]$. Despite all the confusing information on AMT flaps, nowadays 'true' AMT flaps are accepted to base on the unnamed branch of LCFA [32].

The direct cutaneous vessels also arising from the perforator pedicle are the basis of several traditional and the other types of flaps [5]. In general, the blood supply of the AMT flap is based on the cutaneous perforators originating from the medial or lateral margin of the sartorius muscle [4]. The perforators of the AMT flap have been previously determined by several authors $[2,9,11,24,25,29]$. It mainly arises from the LCFA, which has 3 branches: the ascending, the transverse, and the descending [26]. The descending branch has several perforators that have recently been described; however, there is no consensus on their nomenclature $[4,5,27]$. The descending perforators gave out some branches both laterally and medially. The lateral perforators were commonly observed in the middle half of the thigh [4]. The medial perforators were located proximally as fewer in number. The pedicle of the AMT flap, which was firstly described as innominate branch [25], was recently termed as oblique branch, originating from near the base of the LCFA or from the proximal third of the descending branch of the LCFA. Less frequently, the pedicle might originate from the other vessels. The oblique branch terminated as a cutaneous perforator of the AMT skin. This artery was mainly septocutaneous and traversed medially between the overlying rectus femoris and the vastus intermedius muscles, proximally and laterally. It then appeared in the triangle which was formed by the rectus femoris, the sartorius, and the vastus medialis muscles. Along its course on the thigh region, it gave several small branches to the adjacent muscles and emerged from the deep fascia and then supplied the covering skin [4]. Unlike recent publications, these perforators were described in the classical textbooks as superior, middle and inferior groups of the flap pedicle which were named as the fasciocutaneous and the musculocutaneous branches.

The anteromedial free flaps based on the superficial iliac circumflex artery were required for repairing cheek defects, burn scar contractures, traumatic defects of the lower extremity and total facial resurfacing [21]. The local flaps were used for reconstruction of scrotum defect, trochanteric decubitus ulcer, and lower abdominal skin and fascia defects. The authors concluded that the groin flap with AMT extension was a useful modification for repairing the defects [21]. The AMT flaps based on the descending branch of the lateral circumflex femoral vessels provided a long vascular pedicle and a large flap without sacrificing principal vessels and muscles for reconstruction of head and neck region defects following tumour ablation and oromandibular defects [2]. Although, many studies in literature described the advantages of using AMT flaps, there were some studies which reported the disadvantages of these flaps due to possible variations of the LCFA [1, 2]. Notably, several authors controversially discussed vasculature of the thigh region [23]. They noted that possible variants in the vascular anatomy of the region should be examined carefully [22, 23]. Several authors also reported the disadvantages of the AMT such as anatomical variations in the lateral circumflex femoral system and its cutaneous perforators. It was noted that investigators should pay attention on ambiguity of the classification and nomenclature of the arterial tree [20]. For an optimal and safe reconstructive surgery, these types of problems should be eliminated. AMT flap is a fasciocutaneous flap whose blood supply depends on a single, sizeable and consistent cutaneous and perforating branches [27]. A septocutaneous artery pedicle should be usually selected with a diameter of $0.1 \mathrm{~cm}$ or greater when perforating the fascia lata [25]. Similarly, the size of the CB was $0.14 \mathrm{~cm}$ at the level of origin on average in our results. Contrary to the literature reports, we aimed to display anatomical peculiarities of the artery and its muscular, cutaneous, and PBs such as origin, course, and size and to find out and describe the vascular variations of the artery in detail. Two accompanying innominate cutaneous veins and the branches of the anteromedial cutaneous nerve with the $C B$ of the LCFA were determined in all of the examined cases. Thus, we aimed to eliminate disadvantages of the AMT flaps due to vascular differences to prevent complications during flap surgery.

Knowledge of the variation in the course of the artery and cutaneous or perforating branches is of importance for avoiding complications such as bleeding, vessel injuries etc., as well as for understanding anatomical characteristics of the adjacent region while elevating and moving flaps during intraoperative period. Therefore, the localisation, size, length or distance of the surgical reference points of the cutaneous, perforating and MBs were also evaluated in this study. 
Some authors described 3 types of nutrient cutaneous arteries, which emerged from the medial intermuscular space formed by the sartorius, the rectus femoris and vastus medialis muscles. This pedicle supplied to AMT flap as type 1 , the medial branch of the descending branch of the LCFA; type 2 , the superficial femoral artery; and type 3 , the branch of the femoral artery $[5,30]$. The present study differs from the previously published results, since it was observed that the $C B$ emerged from the LCFA in all cases. The AMT flap was a septocutaneous arterial flap based on the septocutaneous perforator originating from the LCFA; the emergence of the septocutaneous perforators was at approximately midthigh in a small triangle representing the juncture of the medial margin of the rectus femoris muscle, the lateral border of sartorius muscle, and the underlying vastus medialis muscle. Similarly, in the present study the flap was shown to be supplied by nutrient cutaneous arteries which emerge from the medial intermuscular space formed by the sartorius, the rectus femoris and vastus medialis muscles in all cases. It was determined that the perforating branch which arose from the LCFA, appeared between middle and lower part of the thigh. Distances between the surgical reference points such as the anterior superior iliac spine, pubic tubercle and interepicondylar line and also the perforating branches were clearly displayed.

The pedicle of the AMT flap is located on average $28.5 \mathrm{~cm}$ distally from the anterior superior iliac spine. While searching for the PB, meticulous dissection is suggested throughout the area on which the flap has been placed. The current study has some limitations related to the fact that the number of cadavers was relatively small. Further detailed anatomical investigations are required in large sample size so as to improve preoperative neurovascular mapping during surgical operations and also to obtain reliable, clear and not confused terminological anatomic data.

\section{CONCLUSIONS}

The results of the present study revealed anatomical features of the anteromedial flaps and provided further information for better understanding of the vascular territory of the anteromedial flaps.

Finally, we conclude that our findings contribute to literature in terms of anatomical knowledge enabling a safe flap surgery.

\section{REFERENCES}

1. Ao M, Asagoe K, Maeta M, Nakagawa F, Saito R, Nagase $Y$ (1998) Combined anterior thigh flaps and vascularised fibular graft for reconstruction of massive composite oromandibular defects. Br J PlastSur, 51: 350-355. doi:10.1054/bjps.1997.0173.

2. Ao $M$, Uno $K$, Maeta $M$, Nakagawa F, Saito $R$, Nagase $Y$ (1999) De-epithelialised anterior (anterolateral and anteromedial) thigh flaps for dead space filling and contour correction in head and neck reconstruction. Br J Plast Surg, 52: 261-267. doi: 10.1054/bjps.1999.3107.

3. Blondeel PN, Van Landuyt KH, Monstrey SJ, Hamdi M, Matton GE, Allen RJ, Dupin C, Feller AM, Koshima I, Kostakoglu N, Wei FC (2003) The "Gent" consensus on perforator flap terminology: preliminary definitions. Plast Reconstr Surg, 112: 1378-1383. doi: 10.1097/01. PRS.0000081071.83805.B6.

4. Cigna E, Chen HC, Ozkan O, Sorvillo V, Maruccia M, Ribuffo D (2014) The anteromedial thigh free flap anatomy: a clinical, anatomical, and cadaveric study. Plast Reconstr Surg, 133: 420-429. doi: 10.1097/01.prs.0000437258.85951.a1.

5. Cormack GC, Lamberty BGH eds. (1994) The arterial anatomy of skin flaps. 2nd Ed. The blood supply to the skin by regions. Elsevier Churchill-Livinstone, pp. 131-275.

6. Har-Shai Y, Hirshowitz B, Marcovich A, Eliachar I, Peretz BA (1984) Blood supply and innervation of the supermedial thigh flap employed in one-stage reconstruction of the scrotum and vulva-an anatomical study. Ann Plast Surg, 13: 504-510.

7. Hupkens P, Van Loon B, Lauret GJ, Kooloos JG, Vehof JW, Hartman EH, Spauwen PH (2010) Anteromedial thigh flaps: an anatomical study to localize and classify anteromedial thigh perforators. Microsurgery, 30: 43-49. doi: 10.1002/micr.20700.

8. Kim JT (2005) New nomenclature concept of perforator flap. Br J Plast Surg, 58: 431-440. doi: 10.1016/j. bjps.2004.12.009.

9. Koshima I, Soeda S, Yamasaki M, Kyou J (1988) The free or pedicled anteromedial thigh flap. Ann Plast Surg, 21: 480-485.

10. Koshima I, Hosoda M, Moriguchi T, Hamanaka T, Kawata S, Hata T (1993a) A combined anterolateral thigh flap, anteromedial thigh flap, and vascularized iliac bone graft for a full-thickness defect of the mental region. Ann Plast Surg, 31: 175-180.

11. Koshima I, Yamamoto H, Hosoda M, Moriguchi T, Orita $Y$, Nagayama H, (1993b) Free combined composite flaps using the lateral circumflex femoral system for repair of massive defects of the head and neck regions: an introduction to the chimeric flap principle. Plast Reconstr Surg, 92: 411-420.

12. Koshima I, Yamamoto H, Moriguchi T, Orita Y (1994) Extended anterior thigh flaps for repair of massive cervical defects involving pharyngoesophagus and skin: an introduction to the "mosaic" flap principle. Ann Plast Surg, 33: 461-462.

13. Koshima I, Hosoda M, Inagawa $K$, Moriguchi T, Orita $Y$ (1996) Free medial thigh perforator-based flaps: New definition of pedicle vessels and versatile application. Ann Plast Surg, 37: 507-515. 
14. Koshima I, Fujitsu M, Ushio S, Sugiyama N, Yamashita S (2005) Flow-through anterior thigh flaps with a short pedicle for reconstruction of lower leg and foot defects. Plast Reconstr Surg, 115: 155-162. doi: 10.1097/01. PRS.0000146870.33661.E7.

15. Leduey A, Leymarie N, Bidault F, Fadel E, Dartevelle P, Kolb F (2013) Cervicothoracic reconstruction with an anteromedial thigh flap: a novel flap concept. J Plast Reconstr Aesthet Surg, 66: 855-858. doi: 10.1016/j.bjps.2012.09.038.

16. Lin CT, Chen SG, Chen TM, Dai NT, Chang SC (2013) Free fasciocutaneous flaps for reconstruction of complete circumferential degloving injury of digits. Microsurgery, 33: 191-197. doi: 10.1002/micr.22064.

17. Matti BA, Crawford BS (1985) A supero-medial thigh flap for urethral reconstruction in epispadias and hypospadias. Br J Plast Surg, 38: 46-50. doi:10.1016/00071226(85)90086-4.

18. McCarthy J ed. (1990) Plastic Surgery. Vol. 3. WB Saunders Company, Philadelphia, USA, p. 376

19. Morimoto K, Furuse K (1988) Surgical treatment for malignants of tissue tumors of the hip. Gan To Kagaku Ryoho, 15 (4 Part 2-3): 1542-1548.

20. Nakagawa F, Maeta M, Uno K, Miyahara T, Saito R (2004) Double anterior (anterolateral and anteromedial) thigh flaps for reconstruction of head and neck defects. Nippon Jibiinkoka Gakkai Kaiho, 107: 645-652.

21. Safak T, Akyurek M (2004) The descending branch of the superficial circumflex artery supplying anteromedial thigh skin. Plast Reconstr Surg, 114: 1118-1122. doi: 10.1097/01.PRS.0000135333.58730.C0.

22. Schoeller T, Shafighi M, Huemer GM, Wechselberger G, Piza-Katzer H (2003) Coverage of pressure sores with free flaps. Chirurg, 74: 671-676. doi:10.1007/s00104003-0668-9.
23. Schoeller T, Huemer GM, Shafighi M, Gurunluoglu R, Wechselberger G, Piza-Katzer H (2004) Free anteromedial thigh flap: clinical application and review of literature. Microsurgery, 24: 43-48. doi:10.1002/micr.10194.

24. Standring S (2005) Gray's anatomy. 39th Ed. Elsevier Churchill-Livingstone, p. 1452.

25. Strauch B, Vasconez LO, Hall-Findlay EJ eds. (1998) Grabb's Encylopedia of flaps, The anteromedial thigh flap. Chapther 41. 2nd Ed. B. Lipincott-Raven, Pheledelphia, pp. 433-438.

26. Song YG, Chen GZ, Song YL (1984) The free thigh flap: a new free flap concept based on the septocutaneous artery. Br J Plast Surg, 37: 149-159.

27. Shimizu T, Fisher DR, Carmichael SW, Bite U (1997) An anatomic comparison of septocutaneous free flaps from the thigh region. Ann Plast Surg, 38: 604-610.

28. Ustüner TE, Mutaf M, Sensöz O (1994) Anteromedialthigh: a source for phallic reconstruction. Ann Plast Surg, 32: 426-430.

29. Visconti G, Salgarello M, Visconti E, Cipriani A, Cina A, Bonomo L (2015) Anatomy of anteromedial thigh perforators: CT-angiography study. Microsurgery, 35: 196-203. doi: 10.1002/micr.22292.

30. Xu Y, Shou K, Zhang Q (1996) Transplantation of the remedial free anteromedial thigh flap. Zhonghua Zheng Xing Shao Shang Wai Ke Za Zhi, 12: 407-409 .

31. Yang JS, Chang M, Li JC (2000) Repairing of inguinal scar contracture deformity in children with transpositional fasciocutaneous flap. Zhongguo Xiu Fu Chong Jian Wai Ke Za Zhi, 14: 361-362.

32. Yu P, Selber J (2011) Perforator patterns of the anteromedial thigh flap. Plast Reconstr Surg, 128: 151e-157e. doi: 10.1097/PRS.0b013e318221dd11. 